\title{
EDITORIAL
}

\section{Current Perspectives on Neuro-Oncology}

While the field of neuro-oncology has made some significant advances in the last several decades, effective treatments for the most aggressive central nervous system (CNS) tumors, particularly malignant glioma, have been elusive. However, as the rapid expansion in molecular biology is changing all areas of oncology at an unprecedented rate, updates such as this issue of Neurotherapeutics can provide a timely snapshot of the most current science and clinical management practices. We would like to formally thank the authors of these articles for contributing their expertise on various subtopics related to brain-tumor therapy, as well as the peer reviewers whose helpful insights served to round out the information presented here. This issue focuses primarily on glioma, but also includes articles on CNS lymphoma, CNS metastases, and pediatric brain tumors to highlight other specific areas of importance in neuro-oncology.

Advances in basic science research have given us a new understanding of the complexity of a disease that has, so far, largely resisted standard therapies and does not respond to treatments that are successful in other solid tumors. Like other medical specialties, neuro-oncology is moving toward developing personalized medicine based on the unique biology of each patient. As we come closer to realizing this goal, the fields of neuroepidemiology and genetics are serving as foundations for devising interventions to target specific abnormalities that give rise to a particular tumor. There is now a concerted national effort to look at risk factors and biomarkers, and this has resulted in increased access to shared databases and tissue samples-a critical step for combating a disease with a relatively low incidence. Molecular techniques for characterizing tumors have revolutionized neuroepidemiology and have become indispensible for refining classification schemes and creating models to predict response to therapies. Epidemiologists are examining individual genetic predisposition not only to developing a certain tumor, but also to tolerance of therapy (toxicity) and to neurocognitive changes.

Similarly, advances in genetics are providing more information on chromosomal abnormalities that may give rise to tumors and serve as targets for therapy. The relatively nascent field of epigenetics is explaining variations in gene expression and has proved especially useful in characterizing low-grade tumors. Low-grade tumors do not have gross chromosomal abnormalities but do have epigenetic abnormalities that can be identified and possibly targeted. The article by Costello and Nagarajan (pp. 436-446) takes us through the most recent developments in a field that is only now being explored for its full potential to reveal mechanisms of tumorigenesis in the brain. Another field focusing on the origin of tumors is that of stem cell biology. The possibility of a neural stem cell progenitor for brain tumors has generated a host of studies that may provide new information on neoplastic transformation and lead to new cell-based and genetic therapies for brain tumors.

To form a comprehensive treatment strategy, however, it is not only important to understand how brain tumors form, but also how they survive and proliferate. Angiogenesis has long been recognized as a process that is critical for survival of highly aggressive tumors, and research into this area continues to provide information on the complex interactions occurring in the tumor microenvironment that give tumors a blood supply to thrive and grow. This research has led to new therapies that are available for clinical use and that are in clinical trials, and the current state of antiangiogenic strategies is presented here by Tate and Aghi (pp. 447-457).

As the biology of brain tumors becomes more defined, parallel strides in clinical management are using these discoveries to enhance treatment. Diagnosing tumors is becoming a more reliable endeavor with advances in imaging methods. Novel magnetic resonance imaging (MRI) techniques are becoming more accurate, noninvasive tools for establishing diagnosis and may be able to delineate features predictive of individual tumor biology. Methods such as MR spectroscopy and diffusionweighted imaging are currently being validated in trials and incorporated into routine clinical use to guide treatment decisions.

The mainstay of treating brain tumors is usually a combination of surgery, radiation therapy, and medical therapy. In the past 25 years, there have been tremendous advances in surgical techniques for brain tumors, resulting in greater extent of resection and lower rates of morbidity and mortality for many tumor types. The discussion on surgery in this issue focuses specifically on the experience with glioma at the University of California, San Francisco. In it, we address one of the most challenging clinical scenarios-when a tumor is in the eloquent cortex related to language-and describe the 
techniques used to preserve function and quality of life while obtaining a maximum safe resection.

Techniques in radiation oncology have likewise evolved to preserve functional areas of brain tissue while increasing doses of radiation to the tumor. In their excellent summary of radiation techniques, Khuntia et al. (pp. 487-499) take us through the transition from classic whole-brain radiation therapy to methods that incorporate imaging, stereotactic radiosurgery, and heavy particles and protons. In devising treatment plans for patients, neuro-oncologists are increasingly interested in finding the most effective combinations of radiation therapy and medical therapy that maximize the effectiveness of each.

Many of the original drugs used for brain tumors were first designed for solid tumors, but pharmaceutical companies are now creating drugs aimed at overcoming obstacles specific to brain tumors. The blood-brain barrier has proven to be a particularly formidable obstacle to getting effective concentrations of drug into the tumor. To overcome this barrier, surgical approaches have been developed that bypass the blood-brain barrier and deliver drugs directly to the tumor. These are being studied in clinical trials and are presented in more detail by Vogelbaum and Bidros (pp. 539-546).

In addition to improved drug-delivery strategies, advances in our understanding of basic biology have resulted in a host of new anticancer drugs. In the 20 years since BCNU (1,3-bis-[2-chloroethyl]-1-nitrosourea) became a standard part of medical management, there has been an explosion of new therapeutic approaches based on biology, including viral and kinase inhibitor therapies. The current status of these strategies is reviewed in this issue.
The continued focus on translational research in the field of neuro-oncology provides a way to move our basic understanding of tumor biology beyond the theoretical and apply it to developing new strategies for eradicating a complex disease. This requires combining techniques and knowledge from a variety of scientific disciplines, as depicted in the montage of images on the cover, which highlights some of the essential components of modern neuro-oncology. As the pace of discovery continues to accelerate at the molecular level, novel treatments are being moved into clinical trials more rapidly than ever and provide hope for patients and physicians fighting brain cancer.

Acknowledgments: We thank Ilona Garner, Department of Neurological Surgery, University of California, San Francisco, for excellent editorial support, and Kenneth Xavier Probst for producing the cover image.

Mitchel S. Berger, M.D.

Kathleen M. Plant Distinguished Professor and Chairman of Neurological Surgery Director, Brain Tumor Research Center University of California, San Francisco

San Francisco, California

Susan M. Chang, M.D.

Professor and Lai Wan Kan Endowed Chair of Neurological Surgery

Director, Division of Neuro-Oncology University of California, San Francisco San Francisco, California 